\title{
Purulent Pericarditis with Concurrent Detection of Streptococcus pneumoniae and Malignant Squamous Cells in Pericardial Fluid
}

\author{
Kensuke Nakazawa ${ }^{1}$, Koji Kanemoto ${ }^{1}$, Hiromichi Suzuki ${ }^{2}$, Michiko Masuda ${ }^{1}$, \\ Yousuke Matsuno ${ }^{1}$, Hiroaki Iijima ${ }^{1}$ and Hiroichi Ishikawa ${ }^{1}$
}

\begin{abstract}
Pneumococcal pericarditis complicated by a malignant effusion has not been reported previously. We experienced an independent 62-year-old man with lung cancer who was hospitalized for acute onset of atrial flutter and moderate pericardial effusion. He was afebrile; however, pericardiocentesis showed Streptococcus pneumoniae and malignant squamous cells in purulent pericardial fluid. This case shows that clinicians should keep in mind the possibility of afebrile bacterial pericarditis in cancer patients with pericardial effusions and that cultures of pericardial fluid should be performed in such patients along with cytological examinations.
\end{abstract}

Key words: pneumococcal pericarditis, Streptococcus pneumonia, pericardial effusion, malignancy, lung cancer

(Intern Med 52: 1413-1416, 2013)

(DOI: 10.2169/internalmedicine.52.9411)

\section{Introduction}

Pericarditis involves inflammation of the pericardium and is a life-threatening disease due to the possible development of cardiac tamponade. Etiologies involving bacteria, viruses, autoimmune diseases, malignancy and idiopathic or viral pathogens are thought to underlie most cases of pericarditis $(1,2)$.

Streptococcus pneumoniae is a common pathogen in patients with pneumonia (3), meningitis (4), sinusitis (5) and otitis media (6). However, it is a rare infectious source in patients with pericarditis, with only case studies of pneumococcal pericarditis having so far been published in recent practice. Only two cases in Japan have been reported in the English literature $(7,8)$. We herein report the case of a patient with lung cancer who developed purulent pericarditis with concurrent detection of $S$. pneumoniae and malignant squamous cells in pericardial fluid, which has not been reported previously.

\section{Case Report}

A 62-year-old man visited our emergency department for acute onset of heart palpitations and a three-day history of appetite loss. He had a long-term history of smoking and had been diagnosed with squamous cell lung cancer with metastases to the hilar and mediastinal lymph nodes and the adrenal gland six months previously. He had declined systemic chemotherapy. Radiotherapy was performed four months earlier; nevertheless, it had been ineffective. The patient regularly visited our clinic; however, he was independent in activities of daily living and did not require support, including nonsteroidal anti-inflammatory drugs, before the onset of symptoms.

In an examination in the emergency department, an electrocardiogram showed atrial flutter and transechocardiography showed a moderate pericardial effusion. The atrial flutter subsided after the initial administration of antiarrhythmic agents, although it recurred the next day and the patient was hospitalized for a further evaluation. On admission, he had

${ }^{1}$ Department of Respiratory Medicine, Tsukuba Medical Center Hospital, Japan and ${ }^{2}$ Department of Clinical Laboratory Medicine, Tsukuba Medical Center Hospital, Japan

Received for publication November 27, 2012; Accepted for publication February 15, 2013

Correspondence to Dr. Hiroichi Ishikawa, hishikawa@tmch.or.jp 


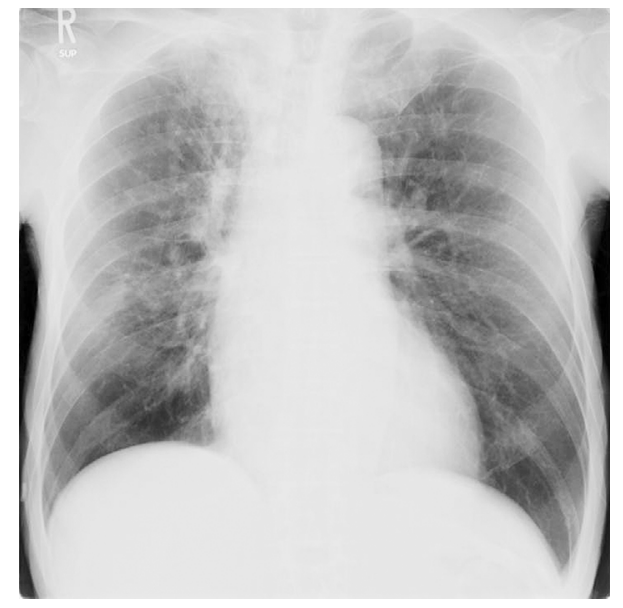

Figure 1. A chest X-ray obtained on day 1 showed a mild increase in the cardiothoracic ratio; however, no new infiltration was observed in either lung field.

tachycardia (143 beats/min) with low blood pressure (92/66 $\mathrm{mmHg}$ ) related to the atrial flutter. His body temperature was $37^{\circ} \mathrm{C}$, his respiratory rate was 20 breaths/min and his oxygen saturation level was $95 \%$ on room air. On physical examination, no heart murmurs, apparent jugular venous distension or peritibial edema were observed. The laboratory findings showed an increased white blood cell (WBC) count $(11,000 / \mu \mathrm{L})$ and an elevated C-reactive protein (CRP) level $(14.0 \mathrm{mg} / \mathrm{dL})$; however, the WBC count $(7,000-8,000 / \mu \mathrm{L})$ and CRP level $(5-8 \mathrm{mg} / \mathrm{dL})$ had been continuously elevated since the diagnosis of lung cancer. A chest X-ray (Fig. 1) did not show any new infiltration.

Digoxin and bisoprolol fumarate were administered for recurrent atrial flutter. Pericardiocentesis was not performed because the pericardial effusion was moderate and there were no signs of bacterial pericarditis. Other diagnostic microbiological analyses, including blood cultures, were not performed at that time. On day 9 after admission, the patient complained of dyspnea and exhibited increases in the WBC count $(16,000 / \mu \mathrm{L})$ and CRP level $(20.6 \mathrm{mg} / \mathrm{dL})$. Urgent enhanced computed tomography did not show any new infiltration in either lung field; however, a prominent effusion was identified in the pericardial space (Fig. 2), and pericardial drainage was promptly performed. The pericardial fluid was purulent with a bloody color, and Gram staining showed the presence of Gram-positive cocci in the fluid. The bacteria were subsequently cultured and identified to be $S$. pneumoniae, which was found to be susceptible to penicillin using the disk dilution method. A cytological examination showed class $\mathrm{V}$, malignant squamous cells. These results were confirmed by two pathologists.

Antimicrobial therapy with tazobactam/piperacillin was administered at a reduced dose (9 g/day) due to the patient's renal impairment along with continuous drainage therapy followed by ampicillin (6 g/day) starting on day 16 . This treatment was effective, and the pericardial catheter was removed after the disappearance of the purulent pericardial fluid. However, the patient's general condition gradually deteriorated due to progressive multiple brain metastases and he died on day 44 after admission.

\section{Discussion}

Fifty years ago, S. pneumoniae was a common pathogen that caused approximately $50 \%$ of cases of bacterial pericarditis (9), most of which were complicated by pneumonia. The development of antimicrobial therapy and increased vaccination for $S$. pneumoniae significantly decreased the incidence of pneumococcal pericarditis, and only 15 cases were reported between 1980 and 1994 (10). Recent surveillance studies of pneumococcal bacteremia identified no patients with pericarditis (11-14), and a large surveillance study found only three cases of pericarditis among 844 patients with pneumococcal bacteremia (15).

Pericardial effusions frequently occur in patients with cancer, particularly in those with breast and lung cancers (16). In such patients, the mechanisms of development of pericardial effusion include direct metastasis to the pericardium, toxicity of chemotherapy or radiation, lymphatic drainage impairment, hypoalbuminemia and opportunistic infections. Malignant or radiation-induced effusions have been identified in most cases $(17,18)$, whereas infection is thought to be a rare etiology of pericardial effusions in cancer patients.

Our case was also initially considered to be malignancyinduced pericarditis with a low possibility of a bacterial origin because the patient had advanced squamous lung cell carcinoma and no fever, apparent pneumonia, empyema or other infective source on an examination performed on admission. In addition, the pericardial effusion was moderate on transechocardiography, and urgent pericardial drainage was not performed because malignant effusions are frequently refractory (16). Therefore, pericardial drainage and subsequent antimicrobial therapy were not administered until one week after admission, when cardiac tamponade occurred. At that time, $S$. pneumoniae and malignant squamous cells were simultaneously identified in the pericardial fluid.

We believe that the pneumococcal pericarditis had been present since admission due to the acute progressive nature of the disease with refractory arrhythmia. However, we cannot exclude the possibility of hospital-onset pneumococcal pericarditis because we did not obtain cultures of the blood or pericardial fluid on admission, the results of which might have led us to an earlier decision to use antimicrobial therapy.

Malignant effusions were also present based on cytology. A few cases of pneumococcal pericarditis in cancer patients have been described $(10,19)$. Simultaneous detection of malignant cells and bacteria in pleural fluid has been reported (20), and cases of bacterial pericarditis with direct invasion of esophageal cancer into the pericardial space have occurred $(21,22)$. However, to the best of our knowledge, there have been no previous reports of concurrent detection 

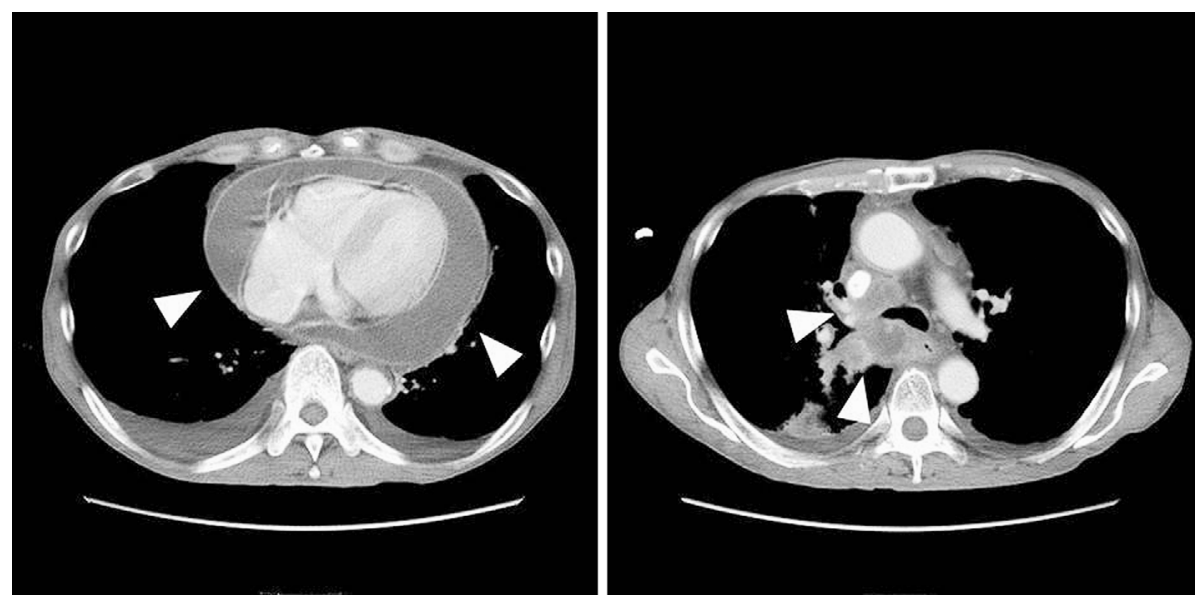

Figure 2. Enhanced chest computed tomography performed on day 9 revealed a prominent pericardial effusion (white arrows on the left image) and enlargement of the metastatic hilar and mediastinal lymph nodes (white arrows on the right image).

of S. pneumoniae and malignant cells in pericardial fluid.

In this case, we were unable to obtain information on the vaccination status of the patient and were unable to preserve the strain. Therefore, we do not know if pneumococcal vaccination might have prevented the pneumococcal pericarditis. In addition, multiple brain metastases were apparent during hospitalization, which led to a fatal outcome, and we also do not know whether prevention or early treatment could have prolonged the patient's survival. However, these treatments may have improved the patient's quality of life. This case serves as a reminder that afebrile bacterial pericarditis can occur in immunocompromised patients and that cultures of pericardial fluid should be performed in such patients along with cytological examinations.

Much of the pathophysiology of pneumococcal pericarditis remains unclear due to the rarity of the condition, and concurrent detection of malignant cells has not been reported previously. The publication of further case reports will provide more clarity on the clinical significance of this disease, including the risk factors and optimal treatments.

The authors state that they have no Conflict of Interest (COI).

\section{References}

1. Levy PY, Corey R, Berger P, et al. Etiologic diagnosis of 204 pericardial effusions. Medicine (Baltimore) 82: 385-391, 2003.

2. Ilan Y, Oren R, Ben-Chetrit E. Acute pericarditis: etiology, treatment and prognosis. A study of 115 patients. Jpn Heart J 32: 315321, 1991.

3. Mandell LA, Wunderink RG, Anzueto A, et al. Infectious Diseases Society of America/American Thoracic Society consensus guidelines on the management of community-acquired pneumonia in adults. Clin Infect Dis 44: S27-S72, 2007.

4. Tunkel AR, Hartman BJ, Kaplan SL, et al. Practice guidelines for the management of bacterial meningitis. Clin Infect Dis 39: 12671284, 2004

5. Sokol W. Epidemiology of sinusitis in the primary care setting: results from the 1999-2000 respiratory surveillance program. Am J Med 111: 19S-24S, 2001.
6. Ruohola A, Meurman O, Nikkari S, et al. Microbiology of acute otitis media in children with tympanostomy tubes: prevalences of bacteria and viruses. Clin Infect Dis 43: 1417-1422, 2006.

7. Nakagawa C, Kasahara K, Yonekawa S, et al. Purulent pericarditis due to Streptococcus pneumoniae diagnosed by pneumococcal urinary antigen assay and $16 \mathrm{~S}$ rDNA sequence of the pericardial fluid. Intern Med 49: 1653-1656, 2010.

8. Nagano N, Yamamoto T, Amano A, Kikuchi K. Infected aneurysm of the aortic arch with purulent pericarditis caused by Streptococcus pneumoniae. Interact Cardiovasc Thorac Surg 10: 459-461, 2010.

9. Klacsmann PG, Bulkley BH, Hutchins GM. The changed spectrum of purulent pericarditis: an 86 year autopsy experience in 200 patients. Am J Med 63: 666-673, 1977.

10. Go C, Asnis DS, Saltzman H. Pneumococcal pericarditis since 1980. Clin Infect Dis 27: 1338-1340, 1998.

11. Suzuki H, Tokuda Y, Shichi D, Ishikawa H, Maeno T, Nakamura H. Morbidity and mortality among newly hospitalized patients with community-acquired pneumococcal bacteremia: A retrospective cohort study in three teaching hospitals in Japan. Geriatr Gerontol Int 2012, DOI: 10.1111/j.1447-0594.2012.00949.x

12. Song JS, Choe PG, Song KH, et al. Risk factors for 30-day mortality in adult patients with pneumococcal bacteraemia, and the impact of antimicrobial resistance on clinical outcomes. Epidemiol Infect 140: 1267-1276, 2011.

13. Giner AM, Kuster SP, Zbinden R, Ruef C, Ledergerber B, Weber R. Initial management of and outcome in patients with pneumococcal bacteremia: a retrospective study at a Swiss university hospital, 2003-2009. Infection 39: 519-526, 2011.

14. Ho PL, Que TL, Ng TK, Chiu SS, Yung RW, Tsang KW. Clinical outcomes of bacteremic pneumococcal infections in an area with high resistance. Eur J Clin Microbiol Infect Dis 25: 323-327, 2006.

15. Kan B, Ries J, Normark BH, et al. Endocarditis and pericarditis complicating pneumococcal bacteraemia, with special reference to the adhesive abilities of pneumococci: results from a prospective study. Clin Microbiol Infect 12: 338-344, 2006.

16. Lestuzzi C. Neoplastic pericardial disease: Old and current strategies for diagnosis and management. World J Cardiol 2: 270-279, 2010.

17. Maisch B, Ristic A, Pankuweit S. Evaluation and management of pericardial effusion in patients with neoplastic disease. Prog Cardiovasc Dis 53: 157-163, 2010.

18. Wilkes JD, Fidias P, Vaickus L, Perez RP. Malignancy-related pericardial effusion. 127 cases from the Roswell Park Cancer In- 
stitute. Cancer 76: 1377-1387, 1995.

19. Gould K, Barnett JA, Sanford JP. Purulent pericarditis in the antibiotic era. Arch Intern Med 134: 923-927, 1974.

20. Czystowska M, Stoklosa A, Szczepulska-Wojcik E, et al. Simultaneous detection of tumor cells and the positive result of genetic test for Mycobacterium tuberculosis in pleural effusion. Pneumonol Alergol Pol 78: 296-301, 2010.
21. Kaufman J, Thongsuwan N, Stern E, Karmy-Jones R. Esophagealpericardial fistula with purulent pericarditis secondary to esophageal carcinoma presenting with tamponade. Ann Thorac Surg 75: 288-289, 2003.

22. Takayama T, Okura Y, Funakoshi K, Sato T, Ohi H, Kato T. Esophageal cancer with an esophagopericardial fistula and purulent pericarditis. Intern Med 52: 243-247, 2013.

(C) 2013 The Japanese Society of Internal Medicine http://www.naika.or.jp/imonline/index.html 